\title{
RARE PRESENTATION OF ADNEXAL MASS: \\ FOLLICULAR DENDRITIC CELL SARCOMA, A CASE REPORT.
}

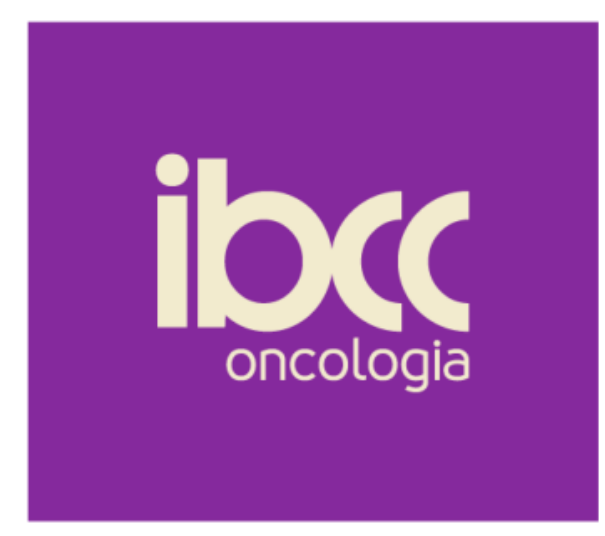

V. Sartorelli ${ }^{1}$, A. Fabiane de Andrade Ferreira Larre ${ }^{1}$, M. Camargo Guimarães Forghieri ${ }^{1}$, L. Leitão ${ }^{1}$, L. Borges de Souza ${ }^{1}$, A.L. Rezende Dias ${ }^{1}$, A. Lopes ${ }^{1}$, G. Lowndes de Souza Pinto ${ }^{1}$, R. Lucio Rangel Costa ${ }^{1}$.

${ }^{1}$ Instituto Brasileiro de Controle do Câncer IBCC, Gynecologic Oncology, Sao Paulo, Brazil.

\section{OBJECTIVES:}

To report a rare histopathological diagnosis of adnexal mass.

\section{METHODS:}

We describe a case of a patient referred to our Gynecology Oncology service to investigate an adnexal mass found in routine exams.

\section{RESULTS:}

A 58-year-old woman was referred to our service due to an adnexal mass associated with hypogastric pain. Physical examination revealed a hardened mass bulging the anteriorright vaginal wall, discreetly painful by the touch. A magnetic resonance imaging (MRI) of the pelvis revealed a right adnexal heterogeneous mass measuring $9,5 \times 4,5 \times 6,5 \mathrm{~cm}$ adjacent to the iliac vessels. There were no altered tumoral markers. She underwent to exploratory laparotomy, and a mass in the right broad ligament was found.

Bilateral salpingoophorectomy and total hysterectomy was performed, as well as the excision of the tumor (which was adhered to the right iliac vein, pelvic wall and obturator fossa) and homolateral pelvic lymphadenectomy. The intraoperatory pathologic evaluation of the lesion suggested a Carcinoma.
The anatomopathologycal study of the retroperitoneal tumor was consistent with poorly differentiated malignant neoplasm. The immunohistochemical analysis showed a strong and diffuse expression of CD23 and negative expression of other markers, ruling out the previous diagnosis and bringing to light the actual histology of the tumor, follicular dendritic cell sarcoma (FDCS).

\section{CONCLUSIONS:}

It is known that FDCS is an uncommon lymphoid neoplasm, especially in pelvic location, with low incidence and indolent growth, difficult to diagnose, but with high rates of local recurrence and eventually distant metastases. There is no standard treatment established and adjuvant therapy is still controversial.

\section{REFERENCES:}

Agaimy, A., Michal, M., et al. Follicular dendritic cell sarcoma: clinicopathologic study of 15 cases with emphasis on novel expression of MDM2, somatostatin receptor 2A, and PD-L1. Annals of Diagnostic Pathology. 2016; 23, 21-28.

Gounder, M., Desai, V., et al. Impact of surgery, radiation and systemic therapy on the outcomes of patients with dendritic cell and histiocytic sarcomas. European Journal of Cancer. 2015; 51(16), 24132422. 